\title{
Effects of anomalous triple-gauge-boson interactions in diboson production with RECOLA2
}

\author{
Mauro Chiesa*, Ansgar Denner \\ Julius-Maximilians-Universität Würzburg, Institut für Theoretische Physik und Astrophysik, \\ D-97074 Würzburg, Germany. \\ E-mail: mauro.chiesa@physik.uni-wuerzburg.de, \\ ansgar.dennerephysik.uni-wuerzburg.de
}

\section{Jean-Nicolas Lang}

Universität Zürich, Physik-Institut, CH-8057 Zürich, Switzerland.

E-mail: jlang@physik.uzh.ch

\begin{abstract}
Diboson production processes are of great importance in high-energy physics and a precise theoretical knowledge of $V V^{(1)}$ production is mandatory not only in view of precision tests of the Standard Model but also in the one of new physics searches. We present results of Ref. [1], where we computed the NLO QCD and NLO electroweak corrections to diboson production processes at the LHC including the effect of the anomalous triple-gauge-boson interactions at NLO QCD accuracy. The anomalous triple-gauge-boson interactions are parametrized in terms of higher-dimensional operators in the effective field theory framework. Our calculation is the first application of RECOLA2 to effective field theory models.
\end{abstract}

Loops and Legs in Quantum Field Theory (LL2018)

29 April 2018 - 04 May 2018

St. Goar, Germany

\footnotetext{
*Speaker.

${ }^{\dagger}$ M. C. would like to thank the organizers for their kind invitation.
} 


\section{Introduction}

Diboson production is of great importance at the LHC. On the one hand, it can be used as a precision test of the Standard Model (SM) and, on the other hand, it is an important source of background both in the context of SM measurements and in the one of New Physics (NP) searches. Moreover, diboson production is sensitive to the gauge-boson self interaction and can be used to set limits on the anomalous triple-gauge-boson interactions.

From the above considerations, it follows that a precise theoretical understanding of this process is mandatory. The current status of the theoretical predictions for diboson production can be summarized as follows. On the QCD side, the two-loop QCD corrections to $\mathrm{pp} \rightarrow \mathrm{VV}^{\prime}(\rightarrow 4 \mathrm{l})$ have been computed, resummed predictions at NNLL+NNLO accuracy and matched predictions at NLO+PS (Parton Shower) accuracy are also available. More recently, an NNLO calculation matched with QCD PS was presented in Ref. [2]. On the electroweak (EW) side, the one-loop EW corrections have been computed for all the four-lepton production processes. We refer to Ref. [1] for a more detailed bibliography.

\section{EFT framework for triple-gauge-boson interaction}

In the literature, the anomalous triple-gauge-boson interaction is usually described in terms of anomalous couplings. More recently have been published results in the SM effective field theory (SMEFT) framework. In the SMEFT, the SM Lagrangian is generalized by adding nonrenormalizable gauge-invariant operators with canonical dimension $D>4$ :

$$
\mathscr{L}^{\mathrm{eff} .}=\mathscr{L}^{\mathrm{SM}}+\sum_{i} \frac{c_{6}^{i}}{\Lambda^{2}} \mathscr{O}_{6}^{i}+\sum_{i} \frac{c_{8}^{i}}{\Lambda^{4}} \mathscr{O}_{8}^{i}+\cdots
$$

In Eq. (2.1) the operators $\mathscr{O}_{D}^{i}$ represent the effect of new physics with a mass scale $\Lambda$ much larger than the electroweak scale and are multiplied by the corresponding Wilson coefficients $c_{D}^{i}$.

We describe the anomalous $W W \gamma$ and $W W Z$ interaction in terms of the following dimension- 6 (Dim-6) operators [3]:

$$
\begin{aligned}
\mathscr{O}_{W W W} & =-\frac{g_{\mathrm{w}}^{3}}{4} \varepsilon_{i j k} W_{\mu \nu}^{i} W^{v \rho j} W_{\rho}^{\mu k}, \\
\mathscr{O}_{W} & =-\mathrm{i} g_{\mathrm{w}}\left(D_{\mu} \Phi\right)^{\dagger} \frac{\tau_{k}}{2} W^{\mu v k}\left(D_{v} \Phi\right), \\
\mathscr{O}_{B} & =+\mathrm{i} \frac{g_{1}}{2}\left(D_{\mu} \Phi\right)^{\dagger} B^{\mu v}\left(D_{v} \Phi\right), \\
\mathscr{O}_{\widetilde{W} W W} & =+\frac{g_{\mathrm{w}}^{3}}{4} \varepsilon_{i j k} \widetilde{W}_{\mu \nu}^{i} W^{v \rho j} W_{\rho}^{\mu k}, \\
\mathscr{O}_{\widetilde{W}} & =+\mathrm{i} g_{\mathrm{w}}\left(D_{\mu} \Phi\right)^{\dagger} \frac{\tau_{k}}{2} \widetilde{W}^{\mu v k}\left(D_{v} \Phi\right),
\end{aligned}
$$

where $g_{\mathrm{w}}=e / s_{\mathrm{w}}, g_{1}=e / c_{\mathrm{w}}, \tau$ are the Pauli matrices and $\Phi$ stands for the Higgs doublet. 
As no Dim-6 operator contributes to the neutral triple-gauge-boson interaction, we consider the following set of Dim-8 operators for the $V V V^{\prime}$ interaction $\left(V, V^{\prime}=Z, \gamma\right)$ [4]:

$$
\begin{aligned}
\mathscr{O}_{B W} & =-\mathrm{i} \Phi^{\dagger} B_{\mu \nu} \frac{\tau_{i}}{2} W^{\mu \rho i}\left\{D_{\rho}, D^{v}\right\} \Phi+\text { h.c. } \\
\mathscr{O}_{W W} & =\mathrm{i} \Phi^{\dagger} \frac{\tau_{i}}{2} \frac{\tau_{j}}{2} W_{\mu \nu}^{i} W^{\mu \rho j}\left\{D_{\rho}, D^{v}\right\} \Phi+\text { h.c. }, \\
\mathscr{O}_{B B} & =\mathrm{i} \Phi^{\dagger} B_{\mu v} B^{\mu \rho}\left\{D_{\rho}, D^{v}\right\} \Phi+\text { h.c. }, \\
\mathscr{O}_{\widetilde{B} W} & =-\mathrm{i} \Phi^{\dagger} \widetilde{B}_{\mu \nu} \frac{\tau_{i}}{2} W^{\mu \rho}\left\{D_{\rho}, D^{v}\right\} \Phi+\text { h.c. },
\end{aligned}
$$

where h.c. denotes the hermitian conjugate, $D_{\mu}$ represents the $\mathrm{SU}(2)_{\mathrm{w}} \times \mathrm{U}(1)_{Y}$ covariant derivative and $\left\{D_{\mu}, D^{v}\right\}=D_{\mu} D^{v}+D^{v} D_{\mu}$.

As a consequence of Eq. (2.1), the cross sections and/or the differential distributions have the form

$$
\sigma=\sigma_{\mathrm{SM}^{2}}+\sigma_{\mathrm{SM} \times \mathrm{EFT} 6}+\sigma_{\mathrm{EFT}^{2}}+\sigma_{\mathrm{SM} \times \mathrm{EFT} 8}+\sigma_{\mathrm{EFT}^{2}}+\ldots
$$

with

$$
\sigma_{\mathrm{SM} \times \mathrm{EFT} 6} \propto \frac{c_{6}}{\Lambda^{2}}, \quad \sigma_{\mathrm{EFT} 6^{2}} \propto \frac{c_{6}^{2}}{\Lambda^{4}} \quad \sigma_{\mathrm{SM} \times \mathrm{EFT} 8} \propto \frac{c_{8}}{\Lambda^{4}}, \quad \sigma_{\mathrm{EFT} 8^{2}} \propto \frac{c_{8}^{2}}{\Lambda^{8}} .
$$

Since the $\sigma_{\mathrm{EFT} 6^{2}}$ and $\sigma_{\mathrm{SM} \times \mathrm{EFT} 8}$ terms are of the same order in the $1 / \Lambda$ expansion, for a generic EFT model a consistent $1 / \Lambda$ expansion should include only the $\sigma_{\mathrm{SM} \times \mathrm{EFT6}}$ contribution if the Dim8 operators are neglected. However, there can be specific models where the $\sigma_{\mathrm{SM} \times \mathrm{EFT} 8}$ term is subleading with respect to the $\sigma_{\mathrm{EFT} 6^{2}}$ one without violating the EFT expansion. For these reasons, in Sect. 4 we present results both with and without the quadratic contribution in the Dim-6 operators. Similar considerations hold for the Dim-8 operators in ZZ production.

The values of the Wilson coefficients that we used in our numerical simulations are consistent with the current experimental limits from the LHC.

\section{Overview of the calculation}

We computed WW $\left(\rightarrow \mathrm{e}^{+} v_{\mathrm{e}} \mu^{-} \bar{v}_{\mu}\right)$, WZ $\left(\rightarrow \mathrm{e}^{+} v_{\mathrm{e}} \mu^{+} \mu^{-}\right)$, and ZZ $\left(\rightarrow \mathrm{e}^{+} \mathrm{e}^{-} \mu^{+} \mu^{-}\right)$production at NLO QCD including the effect of the Dim-6 and Dim-8 operators as described in Sect. 2. For the processes under consideration, also the NLO EW corrections have been calculated. For WW and $\mathrm{ZZ}$ production, we included the effect of the loop-induced $\mathrm{gg} \rightarrow \mathrm{WW}$ and $\mathrm{gg} \rightarrow \mathrm{ZZ}$ processes; though these processes are formally of higher order in $\alpha_{S}$, they can give sizable contributions because of the gluon PDF.

Our calculation relies on the following tools: FEYNRULES, REPT1L, and RECOLA2. We used the MATHEMATICA package FEYNRULES [5, 6] to implement the SM Lagrangian and the relevant Dim-6 and Dim-8 operators. We then employed FEYNRULES to derive the corresponding Feynman rules which were written in a UFO [7] model file.

The PYTHON program REPT1L [8] was used to convert the UFO model file to a RECOLA2 model file. REPT1L performs counterterm expansion, sets up and solves the renormalization conditions and computes the rational terms of type R2 for the model under consideration.

RECOLA2 [8, 9] is an enhanced version of RECOLA [10] employing the tensor-reduction library COLLIER [11] and is used for the automated generation and the numerical evaluation of 


\begin{tabular}{|l|l|l|l|}
\hline Setup & LO $[\mathrm{fb}]$ & NLO QCD $[\mathrm{fb}]$ & NLO EW $[\mathrm{fb}]$ \\
\hline $\mathrm{W}^{+}$Z ATLAS & $18.875(1)_{-6.4 \%}^{+5.2 \%}$ & $34.253(6)_{-4.3 \%}^{+5.3 \%}$ & $17.748(8)_{-6.5 \%}^{+5.3 \%}$ \\
\hline $\mathrm{W}^{+} \mathrm{Z}$ CMS & $14.307(1)_{-6.2 \%}^{+5.0 \%}$ & $26.357(6)_{-4.3 \%}^{+5.4 \%}$ & $13.600(4)_{-6.3 \%}^{+5.1 \%}$ \\
\hline
\end{tabular}

Table 1: Integrated cross section for $\mathrm{WZ}$ production. In the first column $\mathrm{W}^{+} \mathrm{Z}$ is a short-hand notation for the process $\mathrm{pp} \rightarrow \mathrm{e}^{+} v_{\mathrm{e}} \mu^{+} \mu^{-}$. The numbers in parentheses correspond to the statistical error on the last digit. The uncertainties are estimated from the scale dependence.

the tree-level and one-loop amplitudes. While RECOLA is designed for the SM, RECOLA2 relies on external RECOLA2 model files: these model files are user-provided and can be generalizations of the SM.

RECOLA2 was interfaced to a multichannel Monte Carlo integrator that performs all the other steps of the calculation.

\section{Numerical results}

For the process $\mathrm{pp} \rightarrow \mathrm{WZ} \rightarrow \mathrm{e}^{+} v_{\mathrm{e}} \mu^{+} \mu^{-}$we consider two event selections that mimic the ATLAS and CMS analyses of Refs. [12] and [13], respectively. Some results at the cross-section level are collected in Tab. 1. It turns out that the NLO EW corrections are of order $-6 \% /-5 \%$, while the NLO QCD corrections are positive and large $(+80 \% /+90 \%)$ : this is due to the fact that new channels appear at NLO QCD that have gluons in the initial state and are enhanced by the gluon PDF.

Figure 1 shows the differential distributions as a function of the $\mathrm{Z}$ boson $p_{\mathrm{T}}\left(p_{\mathrm{T}, \mu^{+} \mu^{-}}\right)$and of the WZ transverse mass $\left(M_{\mathrm{T}}^{3 l v}\right)$. For these distributions, the NLO EW corrections are negative and their size increases at high $p_{\mathrm{T}}$ and/or $M_{\mathrm{T}}$ up to $-25 \%$ and $-20 \%$ in the tails of the distributions. The NLO QCD corrections are positive and very large, in particular for the $p_{\mathrm{T}, \mu^{+} \mu^{-}}$distribution, where the NLO prediction is almost six times the LO one for $p_{\mathrm{T}, \mu^{+} \mu^{-}} \simeq 600 \mathrm{GeV}$.

Figure 2 shows the ratios

$$
\begin{aligned}
& R_{\text {lin }}^{\mathrm{LO}(\mathrm{NLO})}=\frac{\mathrm{d}\left(\sigma_{\mathrm{SM}^{2}}+\sigma_{\mathrm{SM} \times \mathrm{EFT}}\right)^{\mathrm{LO}(\mathrm{NLO}) \mathrm{QCD}} / \mathrm{d} X}{\mathrm{~d} \sigma_{\mathrm{SM}^{2}}^{\mathrm{LO}(\mathrm{NLO}) \mathrm{QCD}} / \mathrm{d} X}, \\
& R_{\text {quad }}^{\mathrm{LO}(\mathrm{NLO})}=\frac{\mathrm{d}\left(\sigma_{\mathrm{SM}^{2}}+\sigma_{\mathrm{SM} \times \mathrm{EFT}}+\sigma_{\mathrm{EFT}^{2}}\right)^{\mathrm{LO}(\mathrm{NLO}) \mathrm{QCD}} / \mathrm{d} X}{\mathrm{~d} \sigma_{\mathrm{SM}^{2}}^{\mathrm{LO}(\mathrm{NLO}) \mathrm{QCD}} / \mathrm{d} X},
\end{aligned}
$$

with $X=M_{\mathrm{T}}^{3 l v}$. In the two plots, the upper panels contain only the interference terms $\left(\sigma_{\mathrm{SM} \times \mathrm{EFT} 6}\right)$, while in the lower panels also the quadratic terms $\left(\sigma_{\mathrm{EFT} 6^{2}}\right)$ are included. Each line corresponds to a setup where only one of the Wilson coefficients is different from zero. Comparing the predictions for $R_{\text {lin }}^{\mathrm{LO}}$ and $R_{\text {quad }}^{\mathrm{LO}}$ reveals that the largest contribution comes from the quadratic terms (with the only exception of the $c_{W}$ coefficient, where the linear and the quadratic terms tend to compensate). Comparing the left and the right plot in Fig. 2, we conclude that the sensitivity to the Dim-6 

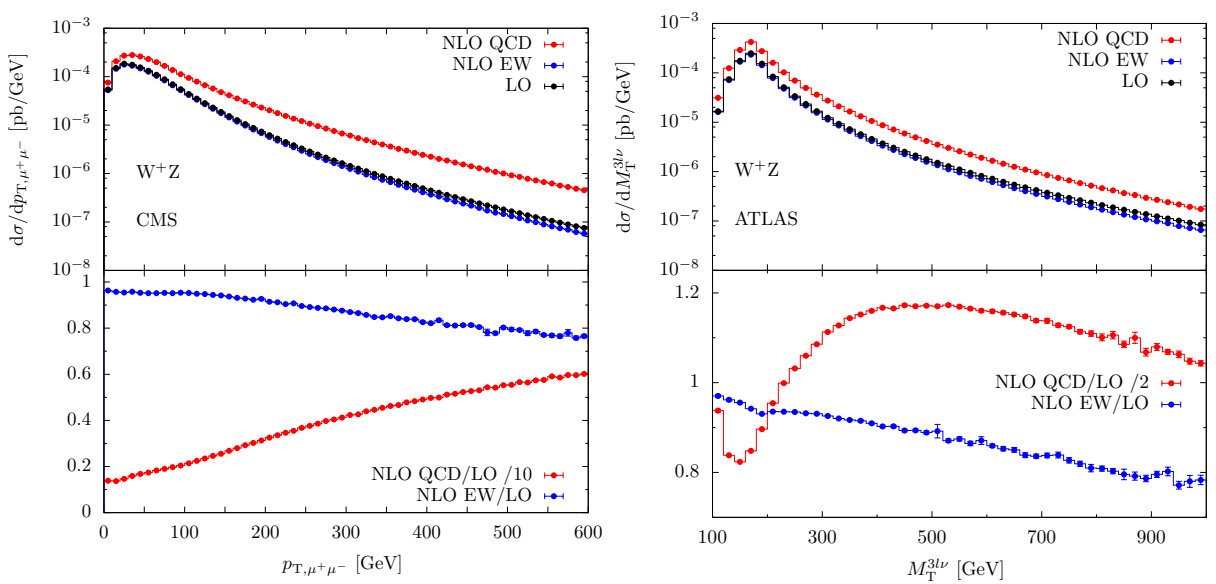

Figure 1: Differential distribution in the transverse momentum of the muon-antimuon pair $\left(p_{\mathrm{T}, \mu^{+} \mu^{-}}\right)$and in the WZ transverse mass $\left(M_{\mathrm{T}}^{3 / v}\right)$ for the process $\mathrm{pp} \rightarrow \mathrm{e}^{+} v_{\mathrm{e}} \mu^{+} \mu^{-}$at $\sqrt{s}=13 \mathrm{TeV}$. The NLO QCD predictions have been divided by a factor 10 (2) in the ratio NLOQCD/LO as a function of $p_{\mathrm{T}, \mu^{+} \mu^{-}}\left(M_{\mathrm{T}}^{3 l v}\right)$.

operators decreases at NLO QCD (with the exception of the $\mathscr{O}_{W W W}$ operator, as already noticed in Ref. [14] for on-shell vector bosons). The reduction in the sensitivity is due to the fact that QCD radiation reduces the centre-of-mass energy of the diboson system with respect to the LO. Since the contribution of the Dim-6 operators increases with the centre-of-mass energy of the diboson system, at NLO QCD the contribution of the Dim-6 operators is suppressed.
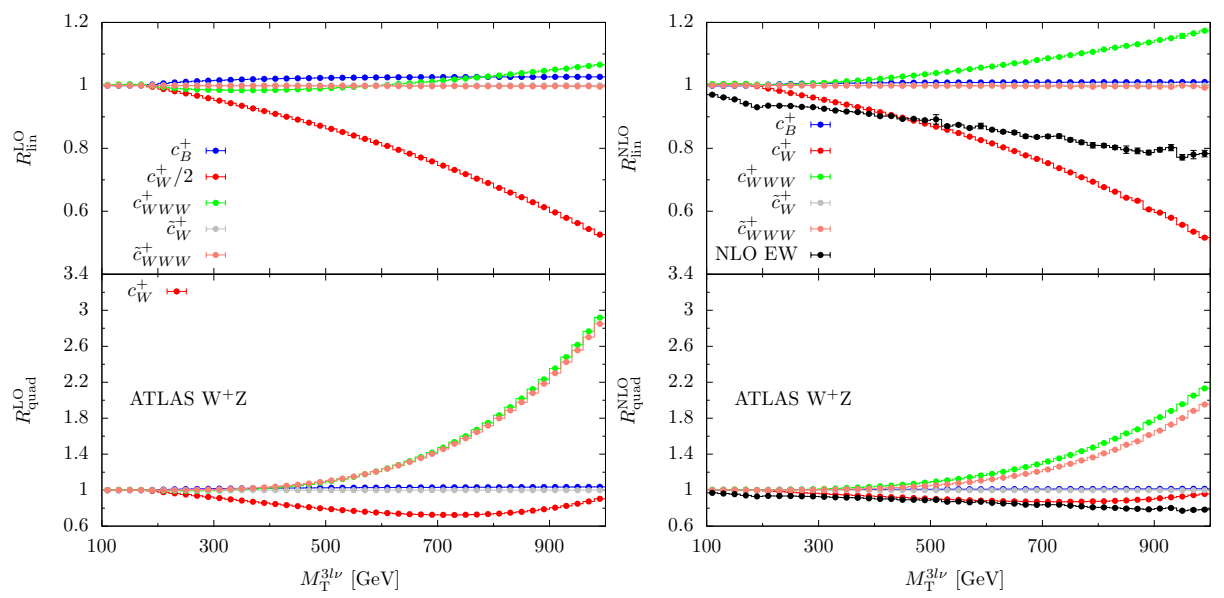

Figure 2: Ratio $R_{\text {lin(quad) }}^{\mathrm{LO}(\mathrm{NLO})}$ as a function of the $\mathrm{WZ}$ transverse mass for the process $\mathrm{pp} \rightarrow \mathrm{e}^{+} v_{\mathrm{e}} \mu^{+} \mu^{-}$. Each line corresponds to a setup where only one of the Wilson coefficients is different from zero. The black lines in the right plot correspond to the NLO EW corrections in the SM. In order to improve the plot readability, in the $R_{\text {lin }}^{\mathrm{LO}}$ ratio (upper panel, left plot) the curve labeled with $c_{W}^{+} / 2$ corresponds to our predictions where the $c_{W}^{+}$coefficient has been divided by a factor 2 .

For the process $\mathrm{pp} \rightarrow \mathrm{ZZ} \rightarrow \mathrm{e}^{+} \mathrm{e}^{-} \mu^{+} \mu^{-}$we consider an event selection that mimics the ATLAS analysis in Ref. [15]. Our predictions at the cross-section level are collected in Tab. 2: the NLO EW corrections are of order $-8 \%$, the NLO QCD corrections are about $+35 \%$, and the con- 


\begin{tabular}{|l|l|l|l|}
\hline LO [fb] & NLO QCD [fb] & NLO EW [fb] & gg [fb] \\
\hline $11.0768(5)_{-7.5 \%}^{+6.3 \%}$ & $14.993(2)_{-2.4 \%}^{+3.1 \%}$ & $10.283(2)_{-7.6 \%}^{+6.4 \%}$ & $1.8584(4)_{-18 \%}^{+25 \%}$ \\
\hline
\end{tabular}

Table 2: Fiducial cross section for the process $\mathrm{pp} \rightarrow \mathrm{e}^{+} \mathrm{e}^{-} \mu^{+} \mu^{-}$at $\sqrt{s}=13 \mathrm{TeV}$. Same notation and conventions as in Tab. 1.

tribution of the loop-induced gg channel is of order $+17 \%$.

Figure 3 shows the differential distributions as a function of the $p_{\mathrm{T}}$ of the hardest $\mathrm{Z}$ boson. $\left(p_{\mathrm{T}, \mathrm{Z}}^{\max }\right)$ and of the four-lepton invariant mass $\left(M_{4 l}^{\mathrm{inv}}\right)$. The NLO EW corrections are negative almost everywhere in the plots and reach the value of $-50 \%$ and $-45 \%$ in the tails of the $p_{\mathrm{T}, \mathrm{Z}}^{\max }$ and $M_{4 l}^{\mathrm{inv}}$ distributions, respectively. The NLO QCD corrections are positive and large, even though not as large as in the case of WZ production. The $M_{4 l}^{\text {inv }}$ distribution is less sensitive to the NLO QCD corrections with respect to the $p_{\mathrm{T}}$ of the hardest $\mathrm{Z}$ boson.
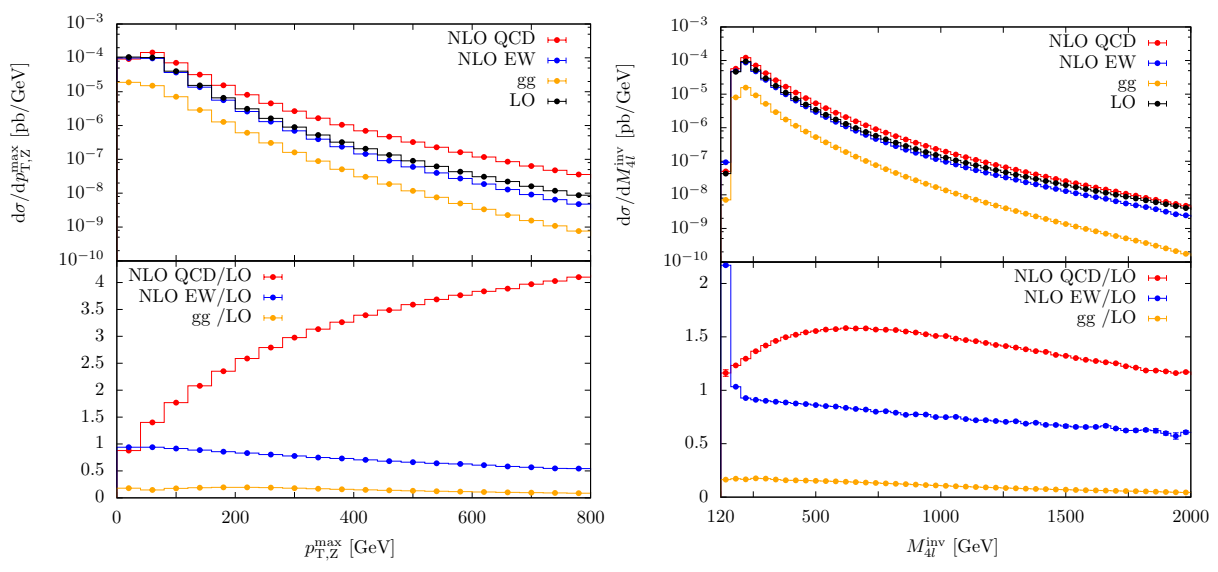

Figure 3: Differential distribution in the hardest Z-boson transverse momentum $\left(p_{\mathrm{T}, Z}^{\max }\right)$ and in the four-lepton invariant mass $\left(M_{4 l}^{\text {inv }}\right)$ for the process $\mathrm{pp} \rightarrow \mathrm{e}^{+} \mathrm{e}^{-} \mu^{+} \mu^{-}$at $\sqrt{s}=13 \mathrm{TeV}$.

The ratios $R_{\text {lin(quad) }}^{\mathrm{LO}(\mathrm{NLO})}$ are shown in Fig. 4 as a function of the $p_{\mathrm{T}}$ of the hardest $\mathrm{Z}$ boson. From the upper panels of Fig. 4 , we notice that $R_{\text {lin }}^{\mathrm{LO}(\mathrm{NLO})}$ is only sensitive to the $c_{\tilde{B} W}$ coefficient, while $R_{\text {quad }}^{\mathrm{LO}(\mathrm{NLO})}$ is sensitive to all the four Dim-8 operators of Eq. (2.3). By comparing $R_{\text {lin }}^{\mathrm{LO}(\mathrm{NLO})}$ and $R_{\text {quad }}^{\mathrm{LO}(\mathrm{NLO})}$ we also conclude that the largest contribution comes from the quadratic term $\left(\sigma_{\mathrm{EFT} 8^{2}}^{\mathrm{LO}(\mathrm{NLO})}\right)$. As in the case of WZ production, the sensitivity to the higher-dimensional operators is reduced at NLO QCD.

\section{Conclusions}

We presented some selected results from Ref. [1], where the processes $\mathrm{pp} \rightarrow \mathrm{e}^{+} v_{\mathrm{e}} \mu^{-} \bar{v}_{\mu}, \mathrm{pp} \rightarrow$ $\mathrm{e}^{+} v_{\mathrm{e}} \mu^{+} \mu^{-}$, and $\mathrm{pp} \rightarrow \mathrm{e}^{+} \mathrm{e}^{-} \mu^{+} \mu^{-}$have been studied in the EFT framework at NLO QCD accuracy. The impact of the Dim-6 operators (Dim-8 operators for ZZ production) has been compared to the NLO QCD and NLO EW corrections in the SM. 

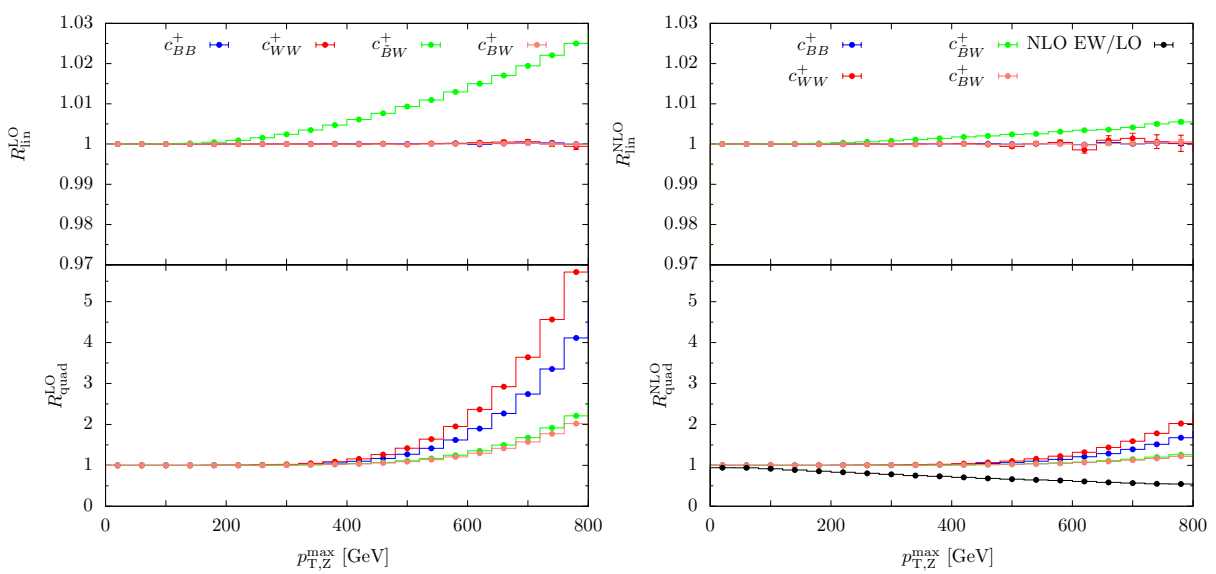

Figure 4: Ratio $R_{\text {lin(quad) }}^{\mathrm{LO}(\mathrm{NLO})}$ as a function of the hardest $\mathrm{Z} p_{\mathrm{T}}$ for the process $\mathrm{pp} \rightarrow \mathrm{e}^{+} \mathrm{e}^{-} \mu^{+} \mu^{-}$. Same notation and conventions as in Fig. 2.

We found that the sensitivity to the anomalous triple-gauge-boson interaction is in general reduced at NLO QCD because of real radiation contributions, which are less sensitive to the anomalous interaction.

Our calculation relies on RECOLA2 for the automated generation and numerical evaluation of the tree-level and one-loop amplitudes. This calculation was the first application of RECOLA2 in the EFT framework.

\section{Acknowledgments}

The work of M.C. and A.D. was supported by the German Science Foundation (DFG) under reference number DE 623/5-1. J.-N. Lang acknowledges support from the Swiss National Science Foundation (SNF) under contract BSCGI0-157722.

\section{References}

[1] M. Chiesa, A. Denner and J. N. Lang, Anomalous triple-gauge-boson interactions in vector-boson pair production with RECOLA2, Eur. Phys. J. C 78 (2018) no.6, 467 [arXiv:1804.01477 [hep-ph]].

[2] E. Re, M. Wiesemann and G. Zanderighi, NNLOPS accurate predictions for $W^{+} W^{-}$production, arXiv: 1805.09857 [hep-ph].

[3] C. Degrande, N. Greiner, W. Kilian, O. Mattelaer, H. Mebane, T. Stelzer, S. Willenbrock and C. Zhang, Effective Field Theory: A Modern Approach to Anomalous Couplings, Annals Phys. 335 (2013) 21 [arXiv:1205.4231 [hep-ph]].

[4] C. Degrande, A basis of dimension-eight operators for anomalous neutral triple gauge boson interactions, JHEP 1402 (2014) 101 [arXiv:1308.6323 [hep-ph]].

[5] N. D. Christensen and C. Duhr, FeynRules - Feynman rules made easy, Comput. Phys. Commun. 180 (2009) 1614 [arXiv:0806.4194 [hep-ph]].

[6] A. Alloul, N. D. Christensen, C. Degrande, C. Duhr and B. Fuks, FeynRules 2.0 - A complete toolbox for tree-level phenomenology, Comput. Phys. Commun. 185 (2014) 2250 [arXiv:1310.1921 [hep-ph]]. 
[7] C. Degrande, C. Duhr, B. Fuks, D. Grellscheid, O. Mattelaer and T. Reiter, UFO - The Universal FeynRules Output, Comput. Phys. Commun. 183 (2012) 1201 [arXiv:1108.2040 [hep-ph]].

[8] A. Denner, J. N. Lang and S. Uccirati, Recola2: REcursive Computation of One-Loop Amplitudes 2, Comput. Phys. Commun. 224 (2018) 346 [arXiv:1711.07388 [hep-ph]].

[9] A. Denner, J. N. Lang and S. Uccirati, NLO electroweak corrections in extended Higgs Sectors with RECOLA2, JHEP 1707 (2017) 087 [arXiv:1705.06053 [hep-ph]].

[10] S. Actis, A. Denner, L. Hofer, J. N. Lang, A. Scharf and S. Uccirati, RECOLA: REcursive Computation of One-Loop Amplitudes, Comput. Phys. Commun. 214 (2017) 140 [arXiv:1605.01090 [hep-ph]].

[11] A. Denner, S. Dittmaier and L. Hofer, Collier: a fortran-based Complex One-Loop LIbrary in Extended Regularizations, Comput. Phys. Commun. 212 (2017) 220 [arXiv:1604.06792 [hep-ph]].

[12] G. Aad et al. [ATLAS Collaboration], Measurements of $W^{ \pm} Z$ production cross sections in pp collisions at $\sqrt{s}=8 \mathrm{TeV}$ with the ATLAS detector and limits on anomalous gauge boson self-couplings, Phys. Rev. D 93, no. 9, 092004 (2016) [arXiv:1603.02151 [hep-ex]].

[13] V. Khachatryan et al. [CMS Collaboration], Measurement of the WZ production cross section in $p p$ collisions at $\sqrt{s}=7$ and $8 \mathrm{TeV}$ and search for anomalous triple gauge couplings at $\sqrt{s}=8 \mathrm{TeV}$, Eur. Phys. J. C 77 (2017) no.4, 236 [arXiv:1609.05721 [hep-ex]].

[14] A. Azatov, J. Elias-Miro, Y. Reyimuaji and E. Venturini, Novel measurements of anomalous triple gauge couplings for the LHC, JHEP 1710 (2017) 027 [arXiv:1707.08060 [hep-ph]].

[15] M. Aaboud et al. [ATLAS Collaboration], Measurement of the ZZ production cross section in proton-proton collisions at $\sqrt{s}=8 \mathrm{TeV}$ using the $\mathrm{ZZ} \rightarrow \ell^{-} \ell^{+} \ell^{\prime-} \ell^{\prime+}$ and $Z Z \rightarrow \ell^{-} \ell^{+} v \bar{v}$ channels with the ATLAS detector, JHEP 1701 (2017) 099 [arXiv:1610.07585 [hep-ex]]. 